\title{
Accuracy of Inchworm Sign on Diffusion-Weighted MRI in Differentiating Muscle-Invasive Bladder Cancer
}

\author{
Huseyin Ozgur Kazan ${ }^{\mathrm{a}, 1, *}$, Meftun Culpan ${ }^{\mathrm{a}, 2}$, Nesrin Gunduz ${ }^{\mathrm{b}, 3}$, Ferhat Keser ${ }^{\mathrm{a}, 4}$, Ayberk Iplikci ${ }^{\mathrm{a}, 5}$, \\ Ramazan Gokhan Atis ${ }^{\mathrm{a}, 6}$ and Asif Yildirim ${ }^{\mathrm{a}, 7}$ \\ ${ }^{a}$ Istanbul Medeniyet University, School of Medicine, Department of Urology, Istanbul, Turkey \\ ${ }^{\mathrm{b}}$ Istanbul Medeniyet University, School of Medicine, Department of Radiology, Istanbul, Turkey
}

Received 3 April 2021

Accepted 6 August 2021

Pre-press 24 August 2021

Published 11 March 2022

\begin{abstract}
.
BACKGROUND: Inchworm sign is a finding on diffusion-weighted magnetic resonance imaging (DWI-MRI) and is used to better stratify T-staging in muscle invasive (MIBC) and non-muscle-invasive bladder cancer (NMIBC). An uninterrupted low submucosal signal on DWI, defined as inchworm sign (IS), indicates NMIBC.

OBJECTIVE: We aimed to define the diagnostic accuracy of IS in primary bladder cancer, as well as find agreement between the urologists and the radiologist.

METHODS: Between December 2018 and December 2020, we retrospectively analyzed 95 primary bladder cancer patients who had undergone multiparametric-MRI before transurethral resection. Patients with former bladder cancer history, tumors smaller than $10 \mathrm{~mm}$, and MRI without proper protocol, as well as patients who did not attend follow-up, were excluded. In total, 71 patients' images were evaluated by a genitourinary specialist radiologist and two urologists. Sensitivity, specificity, positive and negative predictive values of IS and VI-RADS in differentiating MIBC and NMIBC, and interreader agreement between the radiologist and urologists were analyzed.

RESULTS: During follow-up, 38 patients (53.5\%) were IS-positive, while 33 patients (46.5\%) were negative. Among the 33 patients with negative IS, 14 patients (42.4\%) had MIBC. Meanwhile, two out of the 38 IS-positive patients (5.3\%) had MIBC $(p=0.00)$. Sensitivity, specificity, and positive and negative predictive values of IS in predicting MIBC were $87.5 \%$, $63.6 \%, 41.2 \%$ and $94.6 \%$, respectively. The interobserver agreement between the urologists and radiologist was almost perfect $\left(_{K}=0.802\right.$ and $\left._{K}=0.745\right)$
\end{abstract}

CONCLUSION: The absence of IS on DWI is useful in differentiating MIBC from NMIBC. It is a simple finding that can be interpreted by urologists.

Keywords: Diffusion-weighted MRI, inchworm sign, interreader agreement, multiparametric MRI, VI-RADS

\footnotetext{
${ }^{1}$ Huseyin Ozgur Kazan: Orcid ID: 0000-0003-0202-0454

${ }^{2}$ Meftun Culpan: Orcid ID: 0000-0001-8573-1192

${ }^{3}$ Nesrin Gunduz: Orcid ID: 0000-0001-8754-5676

${ }^{4}$ Ferhat Keser: Orcid ID: 0000-0002-2803-6481

${ }^{5}$ Ayberk Iplikci: Orcid ID: 0000-0002-5822-7799

${ }^{6}$ Ramazan Gokhan Atis: Orcid ID: 0000-0002-9065-6104

${ }^{7}$ Asif Yildirim: Orcid ID: 0000-0002-3386-971X
}

${ }^{*}$ Correspondence to: Huseyin Ozgur Kazan, MD, Department of Urology, Istanbul Medeniyet University, School of Medicine, Istanbul, Turkey. Eğitim Mahallesi, Dr. Erkin Cd., 34722 Kadıköy/Istanbul. Tel.: +90 536 2283837; E-mail: ozgur kazan@hotmail.com.; Orcid ID: 0000-0003-0202-0454 


\section{INTRODUCTION}

Multiparametric magnetic resonance imaging (mpMRI) for bladder cancer (BC) is becoming widespread today. Its diagnostic accuracy in optimizing primary tumor staging has been proven by many studies. A meta-analysis by Huang et al. showed that preoperative multiparametric MRI for BC can differentiate between muscle invasive bladder cancer (MIBC) and non-muscle invasive bladder cancer (NMIBC) with a sensitivity of $91 \%$ and a specificity of $96 \%$ [1]. In 2018, Panebianco et al. used the Vesical Imaging Reporting and Data System (VI-RADS) to standardize multiparametric bladder MRI reporting, increase interreader agreement rates, improve sensitivity, and specificity determinig muscle invasion [2]. VI-RADS consists of a scoring system of five points given to T2-weighted MRI, diffusionweighted imaging (DWI), and dynamic contrast enhancement. Recently, some validation studies have been carried out. A recent meta-analysis determined the sensitivity of VI-RADS to be $90 \%$, with a specificity of $90 \%$ [3]. EAU guidelines refer to the high diagnostic accuracy of VI-RADS as needing more validation [4].

Some authors advocate for a combination of T2weighted images and DWI to improve the accuracy of muscle invasion diagnosis. Some authors have found the diagnostic performances of DWI and T2weighted imaging to be similar, although DWI shows a better interobserver agreement and some authors found the sensitivity and specificity of DWI to be similar to VI-RADS [5, 6].

Inchworm sign (IS) was defined by Takeuchi et al. in reference to a finding on a DWI magnetic resonance image. It refers to an uninterrupted low submucosal signal on DWI, made up of a mixture of edematous submucosa, fibrous tissue, capillaries, and inflammatory cells, which indicate NMIBC [7]. Yajima et al. showed that the absence of IS was a significant predictor of the progression of T1-high-grade tumors [8].

In this study, we investigated the diagnostic value of IS in differentiating between MIBC and NMIBC. Our secondary aim was to evaluate the compatibility of DWI-MRI evaluations by urologists compared with radiologists.

\section{MATERIALS AND METHODS}

Between December 2018 and December 2020, we retrospectively analyzed 95 primary $\mathrm{BC}$ patients who underwent DWI-MRI before the transurethral resection of bladder tumor (TUR-BT). Patients with former BC history, tumors smaller than $10 \mathrm{~mm}$, and DWI-MRI without proper protocol, as well as patients who hadn't had a follow-up, were excluded (Fig. 1). Patients without muscularis propria sampling were also not included in our study. Muscularis propria was sampled either at initial TUR-BT, at second TUR-BT, or at cystectomy. In order to optimally evaluate the bladder muscle layer, only primary patients were included in our study. Prior studies mentioned that previous treatments like TUR-BT, intravesical BCG or chemotherapy instillations, and pelvic radiotherapy may cause fibrosis, inflammation, and edema and make it difficult to distinguish between lesions $[9,10]$. Lesions smaller than $10 \mathrm{~mm}$ may not have a stalk yet and have a similar signal intensity in T2 images, which may prevent us from understanding the characteristics of the lesion.

\section{5 primary bladder cancer patients underwent mpMRI}

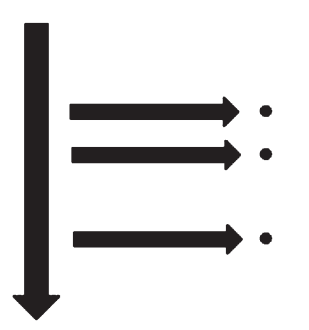

Excluded 10 patients without TUR

Excluded 1 patient without TCC prostate cancer invasion

Excluded 13 patients without proper imaging due to protocol or small, flat tumors

71 patients included

Fig. 1. Patient diagram. 
Since the main evaluation in this study was whether the lesions had a stalk or not, this group of patients was excluded in order to not cause overestimation. In order not to cause bias, TUR-BT was performed by a urologist other than the urologists making the analysis. In total, 71 patients' images were evaluated by a genitourinary specialist radiologist and two urologists, independently.

All patients underwent TUR with monopolar or bipolar electrocautery under white light within two weeks. The specimens were evaluated by an expert uropathologist according to the 2004 WHO/ISUP consensus classification and the TNM classification system. According to EAU guidelines, follow-up with cystoscopy, cytology or second TUR was performed with or without intravesical therapy in accordance with EAU risk groups. After the proper staging of patients with muscle-invasive disease, high-risk NMIBC patients, and those with tumors that could not be completed with TUR-BT, radical cystectomy and pelvic lymph node dissection was performed in eligible patients. Patients with muscle-invasive disease and those suitable for cisplatin received neoadjuvant chemotherapy.

For NMIBC, definitions of recurrence and progression were assessed according to the EORTC and CUETO risk scorings $[11,12]$. Recurrence was assessed as the first recurrence of bladder cancer proven by pathology during follow-up. Progression was assessed as upstaging to MIBC or to a higher disease in the bladder. For MIBC, recurrence was defined as local surgical site recurrence, and progression was defined as a newly diagnosed lymph node or distant metastatis.

While assessing the DWI, three observers evaluated them separately, blind to the pathology reports and to each other. The first observer was a specialist radiologist with ten years of DWI-MRI evaluation experience and two years of VI-RADS assessment experience. The second observer was a urologist with two years of DWI-MRI evaluation experience and five years of TUR experience. The third observer was another urologist with six months of DWI-MRI evaluation experience and two years of TUR experience.

IS was identified according to the criteria defined by Takeuchi et al. [7]. An uninterrupted low submucosal signal, which consisted of edematous tissue, fibrous material, capillaries, and a high signal intensity on the stalk, was determined to be the tumor itself (Fig. 2). A third person, who made the statistical analysis, matched the highest grade tumor in pathology mapping with the reader's assessment of this particular lesion. If one of the multiple tumors in the bladder was pT2, that particular lesion was evaluated in the radiological evaluation. To compare the diagnostic power of IS, we also analyzed the VI-RADS. This was assessed only by the radiologist.

The local ethics committee approved this study (2019/0542). Informed consent was obtained from all individual participants included in the study.

\section{Imaging protocol}

The patients were scanned using the 1.5 Tesla GE Optima MR450w (General Electric, Chicago, Illinois, USA) system. For the purpose of adequate bladder filling and distension, patients were instructed not to void 1-2 hours before imaging and to drink $500-1000 \mathrm{~mL}$ of water in the one hour before the examination. An intramuscular antispasmodic agent was injected to avoid motion and susceptibility artifacts from bowel peristalsis. T2-weighted images were obtained in multiplanar (axial, coronal, and sagittal) reformat. The images were obtained via two-dimensional fast-spin-echo sequences. Diffusion-weighted images were acquired in the axial plane with high $b$ values $(b=0-800-1000$ $1500 \mathrm{~s} / \mathrm{mm} 2)$.

A gadolinium-based contrast agent, adjusted as per body weight $(0.1 \mathrm{mmol} / \mathrm{kg})$, was administered via the antecubital vein using an automatic injector system. Initial contrast enhanced images were acquired $30 \mathrm{~s}$ after the start of injection and repeated four times with the same sequences at every $30 \mathrm{~s}$ to show the early enhancement of the inner layer.

The parameter settings for 1.5 Tesla MRI (including T2-weighted/diffusion-weighted sequences and apparent diffusion coefficient mapping) were as follows: TR values of 3000,7000 , and $6 \mathrm{~ms}$, respectively; TE values of 100,35 , and $1.5 \mathrm{~ms}$, respectively; flip angles of 160, 160, and 12 degrees, respectively; FOV of 24, 26, and $35 \mathrm{~cm}$, respectively; a matrix of $320 \times 320,128 \times 128$, and $288 \times 288$, respectively; slice thickness of 3,4 , and $3 \mathrm{~mm}$, respectively; slice gaps of 1, 1, and $0 \mathrm{~mm}$, respectively; and number of excitations of 2.5, 2-10-10, and 1, respectively. The b values were set at $50,800,1000 \mathrm{~s} / \mathrm{mm} 2$ for DWI.

\section{Statistical analysis}

Associations between IS and demographic/tumor characteristics were analyzed via chi-square tests and independent samples $t$-tests. Bonferroni correction was applied in the presence of three or more variables 

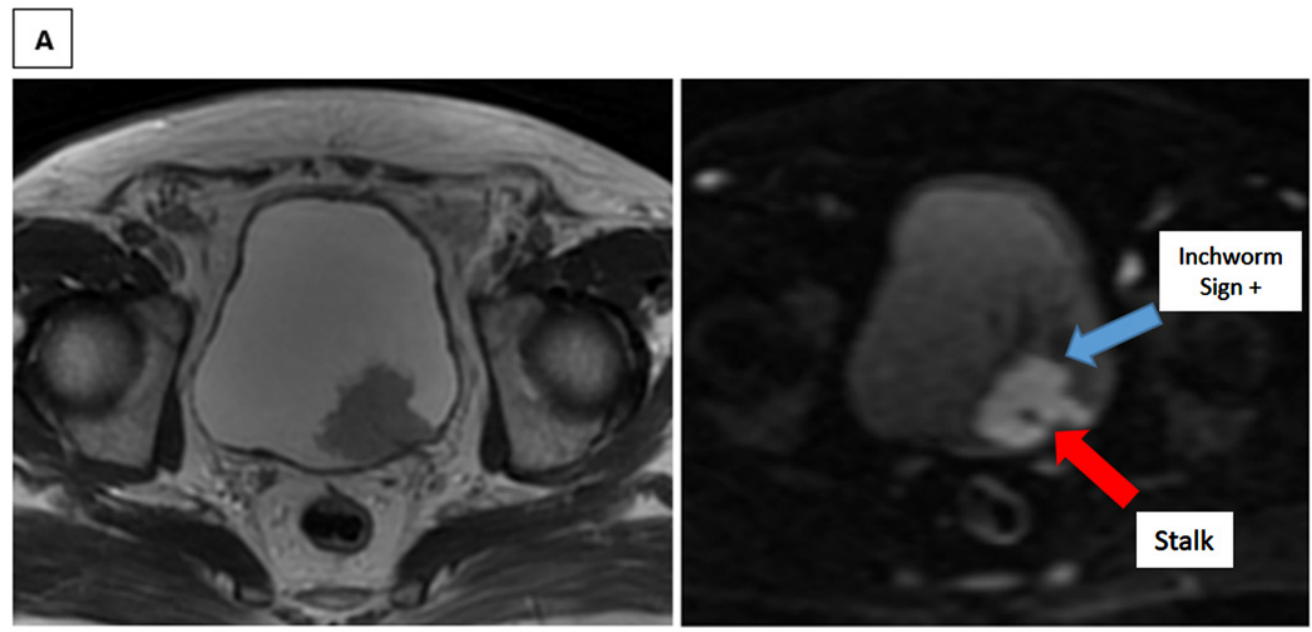

a.

b.
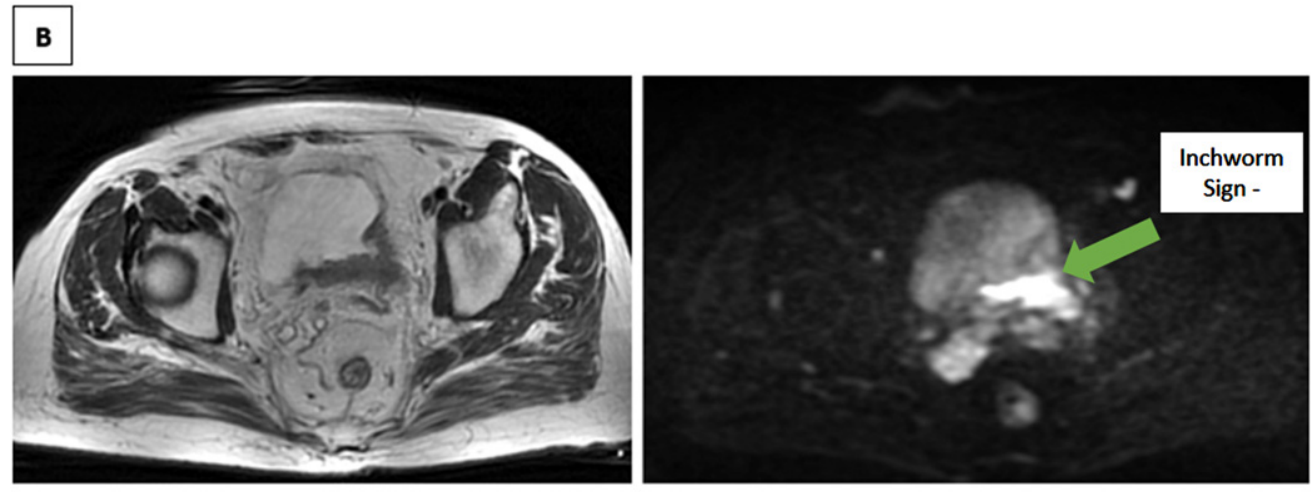

a.

b.

Fig. 2. T2-weighted and Diffusion-weighted Images of patients with and without Inchworm sign. A. Axial MR images of a male patient with a $3 \mathrm{~cm}$ tumor on the posterior bladder wall. a. T2-weighted image b. Diffusion-weighted image tumor with high SI area with a low SI stalk, as an inchworm sign. TUR-BT revealed pT1 high grade papillary urothelial carcinoma without muscle invasion. B. Axial MR images of a male patient with $4.1 \mathrm{~cm}$ tumor on the posterior bladder wall. a. T2- weighted image b. Diffusion-weighted image tumor without inchworm sign. TUR-BT revealed T2 high grade papillary urothelial carcinoma and Radical cystectomy was performed, pathology resulted as pT3 high grade.

in the chi-square test. The interobserver agreement was evaluated by performing a consistency test and calculating the weighted kappa value (Cohen's kappa coefficient). $P$-value $<0.05$ was considered significant. When evaluating the factors affecting the survival Cox regression analysis and evaluating the survival plots, Kaplan Meier analysis was used. These analyses were performed using SPSS (v. 22, IBM).

\section{RESULTS}

\section{Primary outcome: Patients and tumor characteristics}

A total of 71 patients, 38 patients with IS and 33 patients without IS, were included. Both groups had similar demographic characteristics. The mean follow-up time was 10.8 months (Table 1).

Both groups had similar tumor sizes according to radiological measurements (mean $39.9(10-85 \mathrm{~mm})$ vs $30.7 \mathrm{~mm}(10-100 \mathrm{~mm}), p=0.069)$. However, ISnegative tumors were more likely to be solid in structure $(33.3 \%$ vs $10.5 \%, p=0.05)$. Complete TUR-BT was achieved more frequently in the group with positive IS $(84.2 \%$ vs $60.6, p=0.02)$.

The pT stage of initial TUR-BT was seen as $\mathrm{Ta}$ or T1 in most of the IS-positive group tumors (97.4\% vs 63.6\%). Meanwhile, MIBC was seen with a higher rate in the IS-negative group at initial TURBT $(36.4 \%$ vs $2.6 \%, p=0.00)$. At the final stage, pathology muscle invasion was seen in 16 patients, and the majority of them were IS-negative $(42.4 \%$ 
Table 1

Demographic characteristics

\begin{tabular}{|c|c|c|c|c|}
\hline & & $\begin{array}{c}\text { Inchworm Sign - } \\
N=33\end{array}$ & $\begin{array}{c}\text { Inchworm Sign }+ \\
\mathrm{N}=38\end{array}$ & $p$ \\
\hline Age, years & Mean (SD) & $68.1(9.2)$ & $66.8(11.0)$ & $0.610^{\dagger}$ \\
\hline \multicolumn{5}{|l|}{ Gender } \\
\hline Female & & $5(15.2 \%)$ & $4(10.5 \%)$ & \\
\hline Male & & $28(84.8 \%)$ & $34(89.5 \%)$ & $0.559 \ddagger$ \\
\hline \multicolumn{5}{|l|}{ Complaint } \\
\hline Hematuria & & $26(78.8 \%)$ & $35(92.1 \%)$ & \\
\hline LUTS & & $4(12.1 \%)$ & $1(2.6 \%)$ & \\
\hline Flank Pain & & $1(3 \%)$ & $0(0 \%)$ & \\
\hline Insidental & & $2(6.1 \%)$ & $2(5.3 \%)$ & $0.285^{\ddagger}$ \\
\hline BMI, $\mathrm{kg} / \mathrm{m} 2$ & Mean (SD) & $26.9(5.38)$ & $26.6(3.72)$ & $0.780^{\dagger}$ \\
\hline \multicolumn{5}{|l|}{ Smoking history } \\
\hline No & & $8(24.2 \%)$ & $8(21.1 \%)$ & \\
\hline Yes & & $25(75.8 \%)$ & $30(78.9 \%)$ & $0.748^{\ddagger}$ \\
\hline Smoking time, years & Mean (SD) & $31.8(19.4)$ & $34.1(22.9)$ & $0.688^{\dagger}$ \\
\hline \multicolumn{5}{|l|}{ CCI } \\
\hline$\leq 2$ & & $7(21.2 \%)$ & $7(18.4 \%)$ & \\
\hline$>2$ & & $26(78.8 \%)$ & $31(81.6 \%)$ & $0.768^{\ddagger}$ \\
\hline \multicolumn{5}{|c|}{ Family history of cancer } \\
\hline No & & $32(97 \%)$ & $37(97.4 \%)$ & \\
\hline Yes & & $1(3 \%)$ & $1(2.6 \%)$ & $0.919 \ddagger$ \\
\hline Follow-up, months & Mean (SD) & $10.8(5.9)$ & & \\
\hline Min-max & & 2-24 months & & \\
\hline
\end{tabular}

BMI: Body Mass Index, CCI: Charlson Comorbidity Index, ${ }^{\ddagger}:$ Chi-Square Test, ${ }^{\dagger}:$ Independent-Samples $T$ test, SD: Standart Deviation.

vs $5.3 \%, p=0.001)$. The incidence of variant histology was lower in those with IS-positive tumors (7.9\% vs $36.4 \%, p=0.03)$. While eight $(24.2 \%)$ IS-negative patients underwent cystectomy, only two $(5.3 \%)$ patients with IS underwent cystectomy $(p=0.022)$, the group with positive IS received intravesical treatment more frequently $(68.4 \%$ vs $9.1 \%, p=0.00)$ (Table 2).

The diagnostic performance of IS on predicting muscle invasion was shown in Table 3. IS has a sensitivity, specificity, positive predictive value, and negative predictive value of $87.5 \%, 63.6 \%, 41.2 \%$, $94.6 \%$, respectively (Table 3 ).

In the NMIBC group, recurrence and progression were seen more frequently in IS-negative patients (recurrence $=31.6 \%$ vs. $8.3 \%, p=0.027$ and progression $=31.6 \%$ vs. $2.8 \%, p=0.002$ ) (Table 2). In this group, patients with negative IS had worse recurrence-free survival (RFS) than IS-positive patients (2-year RFS: $61.5 \%$ vs $88.4 \%, p=0.016$ ) (Fig. 3). Patients without IS had also worse progression-free survival (PFS) than those with positive IS (2-year PFS: $63.8 \%$ vs. $88.6 \%, p=0.019$ ) (Fig. 4). In NMIBC, factors affecting recurrence-free and progression-free survival were analyzed. IS was found as a factor affecting RFS in the univariate analysis. Gender, tumor size, and variant histology were independent risk factors affecting RFS. Gender, variant histology and IS were found as independent risk factors affecting PFS in NMIBC (Table 5).

\section{Secondary outcome: Interobserver agreement between radiologist and urologists}

When predicting muscle invasion with IS, there are similar results in terms of sensitivity, specificity, positive predictive value, and negative predictive value between the radiologist's analysis and the urologists' analyses. Comparing the results of IS and VI-RADS, VI-RADS had higher sensitivity than IS in terms of predicting muscle invasion; however, both have similar areas under curves ( 0.79 vs. 0.76$)$ (Table 3$)$. Out of 71 patients, the radiologist and first urologist disagreed about seven patients. The kappa coefficient between the radiologist and urologist was 0.802 , which indicates strong agreement. The radiologist and the second urologist also have a high agreement rate $($ kappa $=0.745)($ Table 4$)$.

\section{DISCUSSION}

Muscle invasive and non-muscle invasive $\mathrm{BC}$ have quite different characteristics, especially in terms of prognosis. Proper preoperative staging is essential in 
Table 2

Tumour characteristics

\begin{tabular}{|c|c|c|c|c|}
\hline & & $\begin{array}{c}\text { Inchworm Sign - } \\
\mathrm{N}=33\end{array}$ & $\begin{array}{c}\text { Inchworm Sign + } \\
N=38\end{array}$ & $p$ \\
\hline \multirow[t]{2}{*}{ Tumour size, $\mathrm{mm}$} & Mean (SD) & $39.9(22.5)$ & $30.7(19.5)$ & $0.069^{\dagger}$ \\
\hline & Min-Max & $10-85 \mathrm{~mm}$ & $10-100 \mathrm{~mm}$ & \\
\hline Number of tumours & Mean (SD) & $1.27(0.80)$ & $1.53(1.22)$ & $0.313^{\dagger}$ \\
\hline \multicolumn{5}{|l|}{ Tumour structure } \\
\hline Papillary & & $13(39.4 \%)_{\mathrm{a}}$ & $17(44.7 \%)_{\mathrm{a}}$ & \\
\hline Papillosolid & & $9(27.3 \%)_{a}$ & $17(44.7 \%)_{\mathrm{a}}$ & \\
\hline Solid & & $11(33.3 \%)_{\mathrm{a}}$ & $4(10.5 \%)_{b}$ & $\mathbf{0 . 0 5}$ \\
\hline \multicolumn{5}{|l|}{ Complete TUR-BT } \\
\hline No & & $13(39.4 \%)$ & $6(15.8 \%)$ & \\
\hline Yes & & $20(60.6 \%)$ & $32(84.2 \%)$ & $\mathbf{0 . 0 2} \ddagger$ \\
\hline \multicolumn{5}{|l|}{ pT at initial TUR-BT } \\
\hline $\mathrm{Ta}$ & & $7(21.2 \%)_{\mathrm{a}}$ & $26(68.4 \%)_{\mathrm{b}}$ & \\
\hline $\mathrm{T} 1$ & & $14(42.4 \%)_{\mathrm{a}}$ & $11(28.9 \%)_{\mathrm{a}}$ & \\
\hline $\mathrm{T} 2$ & & $12(36.4 \%)_{\mathrm{a}}$ & $1(2.6 \%)_{b}$ & $\mathbf{0 . 0 0} \ddagger$ \\
\hline \multicolumn{5}{|c|}{ Presence of Muscle at specimen } \\
\hline No & & $13(39.4 \%)$ & $8(21.1 \%)$ & \\
\hline Yes & & $20(60.6 \%)$ & $30(78.9 \%)$ & $0.091^{\ddagger}$ \\
\hline \multicolumn{5}{|l|}{ Grade } \\
\hline Low & & $4(12.1 \%)$ & $10(26.3 \%)$ & \\
\hline High & & $29(87.9 \%)$ & $28(73.7 \%)$ & $0.134 \ddagger$ \\
\hline \multicolumn{5}{|l|}{ Concomitant CIS } \\
\hline No & & $25(75.8 \%)$ & $34(89.5 \%)$ & \\
\hline Yes & & $8(24.2 \%)$ & $4(10.5 \%)$ & $0.124^{\ddagger}$ \\
\hline \multicolumn{5}{|l|}{ Variant histology } \\
\hline No & & $21(63.6 \%)$ & $35(92.1 \%)$ & \\
\hline Yes & & $12(36.4 \%)$ & $3(7.9 \%)$ & $\mathbf{0 . 0 3} \ddagger$ \\
\hline \multicolumn{5}{|l|}{ Second TUR-BT } \\
\hline No & & $23(69.7 \%)$ & $28(73.7 \%)$ & \\
\hline Yes & & $10(30.3 \%)$ & $10(26.3 \%)$ & $0.710^{\ddagger}$ \\
\hline \multicolumn{5}{|l|}{ pT at second TUR-BT } \\
\hline No residual tumour & & $1(10 \%)_{\mathrm{a}}$ & $4(40 \%)_{a}$ & \\
\hline CIS & & $1(10 \%)_{\mathrm{a}}$ & $0(0 \%)_{a}$ & \\
\hline $\mathrm{Ta}$ & & $1(10 \%)_{\mathrm{a}}$ & $3(30 \%)_{a}$ & \\
\hline $\mathrm{T} 1$ & & $3(30 \%)_{\mathrm{a}}$ & $3(30 \%)_{\mathrm{a}}$ & \\
\hline $\mathrm{T} 2$ & & $4(40 \%)_{\mathrm{a}}$ & $0(0 \%)_{\mathrm{b}}$ & $0.099^{\ddagger}$ \\
\hline \multicolumn{5}{|l|}{ Intravesical therapy } \\
\hline None & & $30(90.9 \%)$ & $12(31.6 \%)$ & \\
\hline Mitomycin-C & & $2(6.1 \%)$ & $6(15.8 \%)$ & \\
\hline BCG & & $1(3 \%)$ & $20(52.6 \%)$ & $\mathbf{0 . 0 0 0}$ \\
\hline \multicolumn{5}{|l|}{ Cystectomy } \\
\hline No & & $25(75.8 \%)$ & $36(94.7 \%)$ & \\
\hline Yes & & $8(24.2 \%)$ & $2(5.3 \%)$ & $\mathbf{0 . 0 2 2} \ddagger$ \\
\hline \multicolumn{5}{|c|}{ Muscle Invasion at final pathology } \\
\hline No & & $19(57.6 \%)$ & $36(94.7 \%)$ & \\
\hline Yes & & $14(42.4 \%)$ & $2(5.3 \%)$ & $0.001^{\ddagger}$ \\
\hline \multicolumn{5}{|l|}{ Recurrence } \\
\hline \multicolumn{5}{|c|}{ Non-Muscle Invasive BC } \\
\hline Non-Recurrent & & $13(68.4 \%)$ & $33(91.7 \%)$ & \\
\hline Recurrent & & $6(31.6 \%)$ & $3(8.3 \%)$ & $\mathbf{0 . 0 2 7}$ \\
\hline \multicolumn{5}{|l|}{ Muscle Invasive BC } \\
\hline Non-Recurrent & & $14(100 \%)$ & $1(50 \%)$ & \\
\hline Recurrent & & $0(0 \%)$ & $1(50 \%)$ & $0.125^{\mathrm{f}}$ \\
\hline \multicolumn{5}{|l|}{ Progression } \\
\hline Non-Muscle Invasiv & & & & \\
\hline Non-Progressed & & $13(68.4 \%)$ & $35(97.2 \%)$ & \\
\hline Progressed & & $6(31.6 \%)$ & $1(2.8 \%)$ & $\mathbf{0 . 0 0 2} \ddagger$ \\
\hline Muscle Invasive BC & & & & \\
\hline Non-Progressed & & $13(92.9 \%)$ & $1(50 \%)$ & \\
\hline Progressed & & $1(7.1 \%)$ & $1(50 \%)$ & $0.242^{\mathrm{f}}$ \\
\hline
\end{tabular}

${ }^{\dagger}$ : Independent-Samples $T$ test, ${ }^{\ddagger}$ : Chi-Square Test, ${ }^{\text {f }}$ : Fisher’s Exact Test, SD: Standart Deviation. 
Table 3

Inchworm sign and VI-RADS on predicting muscle invasive bladder cancer

\begin{tabular}{lccccc}
\hline $\begin{array}{l}\text { Reader: } \\
\text { Evaluation: }\end{array}$ & $\begin{array}{c}\text { Radiologist } \\
\text { Inchworm Sign }\end{array}$ & $\begin{array}{c}\text { 1st Urologist } \\
\text { Inchworm Sign }\end{array}$ & $\begin{array}{c}\text { 2nd Urologist } \\
\text { Inchworm Sign }\end{array}$ & $\begin{array}{c}\text { Radiologist } \\
\text { VI-RADS } \geq 3\end{array}$ & $\begin{array}{c}\text { Radiologist } \\
\text { VI-RADS } \geq 4\end{array}$ \\
\hline Sensitivity & $87.5 \%$ & $87.5 \%$ & $81.3 \%$ & $93.8 \%$ & $93.8 \%$ \\
Specificity & $63.6 \%$ & $65.5 \%$ & $67.3 \%$ & $40 \%$ & $63.6 \%$ \\
PPV & $41.2 \%$ & $42.4 \%$ & $41.9 \%$ & $31.3 \%$ & $42.9 \%$ \\
NPV & $94.6 \%$ & $94.7 \%$ & $92.5 \%$ & $95.7 \%$ & $97.2 \%$ \\
AUC & 0.76 & 0.76 & 0.74 & 0.67 & 0.79 \\
\hline
\end{tabular}

AUC: Area under curve, NPV: Negative predictive value, PPV: Positive predictive value.

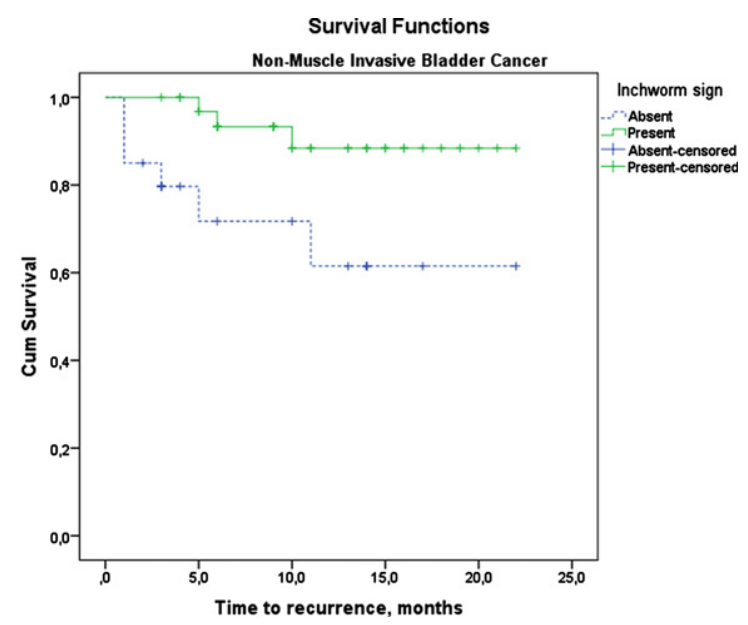

Fig. 3. Recurrence-free Kaplan-Meier Survival Analysis of NonMuscle Invasive Bladder Cancer patients.

Table 4

Interobserver agreement between radiologist and urologists

\begin{tabular}{lcc}
\hline & 1st Urologist & 2nd Urologist \\
\hline Radiologist & $\mathrm{K}=0.802$ & $\mathrm{~K}=0.745$ \\
& Disagreed patients $=7$ & $\begin{array}{c}\text { Disagreed patients }=9 \\
\mathrm{~K}=0.829 \\
\text { 1st Urologist }\end{array}$ \\
& & Disagreed patients $=6$ \\
\hline
\end{tabular}

K: Kappa Coefficient.

primary $\mathrm{BC}$ and is becoming more and more important. Recently, with the introduction of VI-RADS gradually taking place in the proper and standardized staging, MRI has started to play an important role in the local staging of primary BC. In this study, we found that the evaluation of IS in DWI has a high diagnostic value in determining muscle invasive disease in primary BC. It is very important to predict muscle invasive disease only with IS without the need for detailed scoring. In addition, with the VI-RADS evaluation, IS was shown to have very similar diagnostic values (sensitivity $=93.8 \%$ vs. $87.5 \%$, specificity $=63.6 \%$ vs $63.6 \%$ ).

IS was first used by Takeuchi et al. to differentiate MIBC from NMIBC with DWI-MRI. IS was

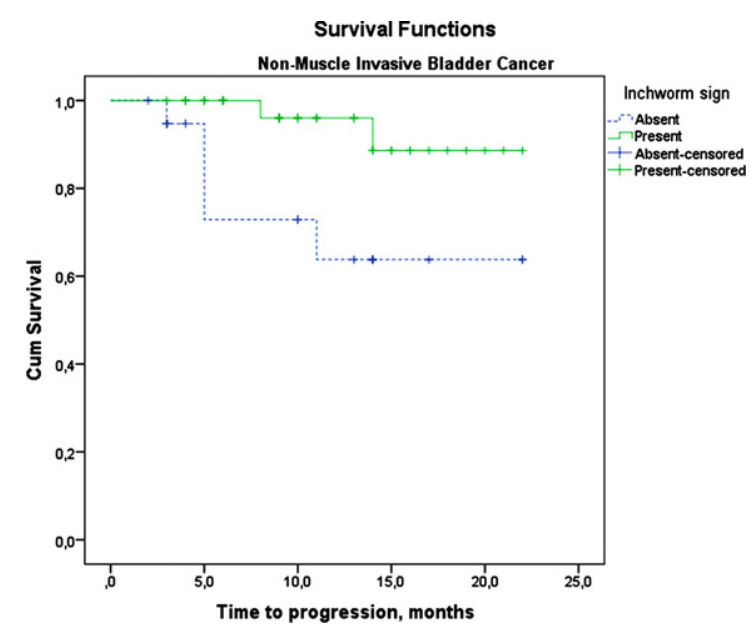

Fig. 4. Progression-free Kaplan-Meier Survival Analysis of NonMuscle Invasive Bladder Cancer patients.

described as a high signal intensity (SI) tumor with a low signal intensity submucosal stalk or a thickened submucosa, which is an archlike shape similar to an inchworm. A histopathological analysis of the submucosal stalk, which has low SI corresponding to the tumor, revealed smooth muscle, capillaries, and edematous tissue. Saito et al. also analyzed the submucosal stalk histopathology and found edema, inflammatory cells, capillaries, and fibrous connective tissue [13]. Takeuchi et al. found that the accuracy of T2-weighted images differentiating T2-T4 tumors from Tis-T1 tumors increased from $79 \%$ to $96 \%$ when DWI was added. Moreover, the interobserver agreement between radiologists increased from $70 \%$ to $88 \%$ [7].

The current study showed that MIBC could be differentiated from NMIBC with great accuracy only by evaluating IS on DWI-MRI. In our study, sensitivity and specificity were $87.5 \%$ and $63.6 \%$, respectively. Huang et al. reviewed 17 studies in a meta-analysis and found the sensitivity and specificity of DWIMRI to be $86 \%$ and $92 \%$ at differentiating stage $\mathrm{T} 2$ or higher from, T1 or lower tumors [1]. The 
Table 5

Factors affecting recurrence-free and progression-free survivals in non-muscle invasive bladder cancer

\begin{tabular}{|c|c|c|c|c|}
\hline \multirow[b]{2}{*}{ Recurrence-free Survival } & \multicolumn{2}{|c|}{$\begin{array}{l}\text { Univariate logistic } \\
\text { regression analysis }\end{array}$} & \multicolumn{2}{|c|}{$\begin{array}{l}\text { Multivariate logistic } \\
\text { regression analysis }\end{array}$} \\
\hline & $p$ & $\mathrm{HR}(\% 95 \mathrm{CI})$ & $p$ & $\mathrm{HR}(\% 95 \mathrm{CI})$ \\
\hline Gender ${ }^{\#}$ & $0,008^{*}$ & $0.167(0.044-0.630)$ & $0.016^{*}$ & $0.029(0.002-0.519)$ \\
\hline BMI & 0.263 & $1.066(0.954-1.191)$ & & \\
\hline Tumor size & $0.001^{*}$ & 1.029 (1.016-1.043) & $0.018^{*}$ & $1.047(1.008-1.087)$ \\
\hline Tumor multiplicity & 0.765 & $0.943(0.642-1.380)$ & & \\
\hline pT Stage & $0.012^{*}$ & $14.495(1.805-116.4)$ & & \\
\hline Pathology Grade & 0.267 & $3.261(0.405-26.245)$ & & \\
\hline Carcinoma in-situ & 0.321 & $2.220(0.460-10.72)$ & & \\
\hline Variant tumor & $0.025^{*}$ & 5.335 (1.239-22.97) & $0.023^{*}$ & $224.87(2.1-24097.9)$ \\
\hline Intravesical treatment & $0.045^{*}$ & $0.178(0.033-0.97)$ & & \\
\hline Inchworm sign & $0.036^{*}$ & $0.225(0.056-0.906)$ & & \\
\hline \multicolumn{5}{|l|}{ Progression-free Survival } \\
\hline Gender ${ }^{\#}$ & $0.021^{*}$ & $0.165(0.036-0.766)$ & $0.014^{*}$ & $0.105(0.017-0.638)$ \\
\hline BMI & 0.554 & $1.043(0.907-1.198)$ & & \\
\hline Tumor size & 0.135 & $1.011(0.997-1.025)$ & & \\
\hline Tumor multiplicity & 0.784 & $0.942(0.613-1.440)$ & & \\
\hline pT Stage & 0.117 & $154.35(0.3-83592.8)$ & & \\
\hline Pathology Grade & 0.266 & $37.2(0.06-21728.9)$ & & \\
\hline Carcinoma in-situ & 0.453 & $0.039(0.00-191.2)$ & & \\
\hline Variant tumor & $0.001^{*}$ & $10.79(2.59-44.83)$ & $0.002^{*}$ & $27.54(3.542-214.14)$ \\
\hline Intravesical treatment & 0.123 & $0.070(0.002-2.053)$ & & \\
\hline Inchworm sign & $0.02^{*}$ & $0.149(0.03-0.739)$ & $0.015^{*}$ & $0.094(0.014-0.632)$ \\
\hline
\end{tabular}

Cox-Regression Analysis. ${ }^{*}: p<0.05$, ${ }^{\#}$ : Male is the indicating value.

recently proposed VI-RADS for multiparametric bladder MRI is obtained by scoring T2-weighted, diffusion-weighted (DW), and dynamic contrastenhanced (DCE) sequences on a 5-point Likert scale. Panebianco et al. defined VI-RADS; according to their classification, tumors with a stalk were considered DWI group 1 and 2, which were considered IS in our study [2]. Various studies showed heterogeneous sensitivities and specificities in terms of differentiating MIBC from NMIBC. Sensitivity values vary between $78 \%$ and $95 \%$, while specificity varies between $44 \% 96 \%$ [6, 14-19]. Some of these authors evaluated each MRI sequence separately. Wang et al. and Arita et al. showed that DWI has the same sensitivity and specificity as VI-RADS [6, 19]. Makboul et al. found lower sensitivity of DWI, but a similar specificity to VI-RADS [15]. In our study, VI-RADS had slightly higher sensitivity than IS, and they had similar specificities. The area under the curves were also quite similar to each other, when 4 is used as the cut-off point. One of the limitations of VI-RADS, as stated by the EAU, is that there is no consensus regarding the cut-off value. Therefore, each study analyzed both cut-off values. Our sensitivity results of DWI and final VI-RADS evaluation were similar to other studies; however, specificity was lower. Four out of six studies had a specificity higher than 0.80 , while one study had a specificity of 0.44 .
It is a fact that these studies are very valuable in terms of developing standards for bladder multiparametric MRI, and more homogeneous results will occur with the expansion of the literature. It is an important advantage that IS has similar results with VI-RADS and can be evaluated more quickly without the need for additional scoring. IS will be very advantageous in terms of its use in clinical practice.

In our study, NMIBC patients without IS had higher recurrence and progression rates. The study by Yajima et al. evaluated the effect of IS on the recurrence or progression of pT1 bladder tumors. They found that the absence of IS is an independent risk factor for progression, but has no impact on recurrence. In our study, NMIBC patients without IS had worse 2-year RFS $(61.5 \%$ vs $88.4 \%$, $p=0.016)$ and PFS (63.8\% vs. $88.6 \%, p=0.019)$. In the regression analysis, IS was found to be an independent risk factor affecting PFS. In the univariate analysis, IS was also a factor affecting RFS in univariate analysis. We showed that IS-negative patients have worse prognostic features in terms of both recurrence and progression compared to positive patients.

Yajima et al. found that tumors without IS had a higher rate of non-papillary tumor structure. In our study, we obtained a similar result: the accompaniment of a solid tumor structure and the absence of 
IS was more frequent [8]. Another finding observed in our study was the relationship between incomplete TUR rates and the absence of IS. This can be explained by the fact that tumors without IS often have irregular margins and are more invasive tumors. Variant histology should be evaluated in the same way, as it is known that tumors with variant histology are more commonly encountered as muscle invasive disease.

The interreader agreement between the radiologist and the urologist in our study showed an "almost perfect" agreement, which was calculated through Cohen's kappa coefficient $(\mathrm{K}=0.802)$. Another and less experienced urologist had substantial agreement with the radiologist $(\mathrm{K}=0.745)$. Yajima et al. found excellent agreement between a radiologist and a urologist in defining IS $(K=0.95)$, without specifying sensitivity and specificity of muscle invasion [8]. Kobayashi et al. found an excellent agreement for DWI-MRI between two radiologists $(\mathrm{K}=0.88)$; however, there was only moderate agreement for T2-weighted sequences $(\mathrm{K}=0.67)$ [5]. Arita et al. evaluated the interreader agreement of VI-RADS and its sequences between two radiologists and two urologists. Between the urologists and radiologists, agreement for DWI-MRI was higher than agreement for T2-weighted and DCE-MRI ${ }_{K}=0.88$ vs 0.80 and $0.70)$ and equal to VI-RADS [19].

IS is a simple finding on DWI-MRI that may provide urologists means to interpret the primary staging. The strength of our study is that it shows the similar diagnostic power of IS with VI-RADS and the diagnostic accuracy of urologists in terms of muscle invasion using only a simple IS in patients with primary bladder tumors.

However, there are several limitations to our study. It used a small sample size, especially regarding patients who underwent cystectomy. Some of the patients who underwent cystectomy received neoadjuvant chemotherapy, meaning cystectomy pathology was not included for them. Depending on the clinical approach and certain contraindications, patients had to receive different treatments such as intravesical treatments during patient management. The ideal interobserver variability is evaluated through the same disciplines, but the secondary aim of this study was to assess agreement between urologists and radiologists in terms of IS. Therefore, we evaluated the compatibility between a radiologist and urologists with different levels of experience. We also analyzed recurrence and progression, though the follow-up period was short.

\section{CONCLUSION}

IS on DWI-MRI is simple and accurate in terms of differentiating NMBIC from MIBC according to both radiologists and urologists.

\section{ACKNOWLEDGMENTS}

The authors have no acknowledgments

\section{FUNDING}

The authors report no funding.

\section{AUTHOR CONTRIBUTIONS}

All authors contributed to the study conception and design. Interpretation of data was performed by [Huseyin Ozgur Kazan], [Meftun Culpan], [Nesrin Gunduz], [Ferhat Keser] [Ayberk Iplikci], [Ramazan Gokhan Atis], [Asif Yildirim]. The first draft of the manuscript was written by [Huseyin Ozgur Kazan], [Meftun Culpan] and all authors commented on previous versions of the manuscript. Final version of the manuscript was written by [Huseyin Ozgur Kazan], [Meftun Culpan], [Nesrin Gunduz], [Ramazan Gokhan Atis], [Asif Yildirim]. All authors had access to the data, read and approved the final manuscript.

\section{CONFLICT OF INTEREST}

The authors, Kazan HO., Culpan M., Gunduz N., Keser F., Iplikci A., Atis RG., Yildirim A. declare that they have no conflict of interest.

\section{REFERENCES}

[1] Huang L, Kong Q, Liu Z, Wang J, Kang Z, Zhu Y. The Diagnostic Value of MR Imaging in Differentiating T Staging of Bladder Cancer: Radiology. 2018;286(2).

[2] Panebianco V, Narumi Y, Altun E, Bochner BH, Efstathiou JA, Hafeez S, et al. Multiparametric Magnetic Resonance Imaging for Bladder Cancer: Development of VI-RADS (Vesical Imaging-Reporting And Data System). Eur Urol. 2018;74(3):294-306.

[3] Luo C, Huang B, Wu Y, Chen J, Chen L. Use of Vesical Imaging-Reporting and Data System (VI-RADS) for detecting the muscle invasion of bladder cancer: a diagnostic meta-analysis. Eur Radiol. 2020;30(8):4606-14.

[4] Witjes JA, Bruins HM, Cathomas R, Compérat EM, Cowan NC, Gakis G, et al. European Association of Urology Guidelines on Muscle-invasive and Metastatic Bladder Cancer: Summary of the 2020 Guidelines. Eur Urol [Internet]. 2021;79(1):82-104. Available from: https://linkinghub. elsevier.com/retrieve/pii/S030228382030230X 
[5] Kobayashi S, Koga F, Yoshida S, Masuda H, Ishii C, Tanaka $\mathrm{H}$, et al. Diagnostic performance of diffusion-weighted magnetic resonance imaging in bladder cancer: potential utility of apparent diffusion coefficient values as a biomarker to predict clinical aggressiveness. Eur Radiol. 2011;21(10): 2178-86.

[6] Wang H, Luo C, Zhang F, Guan J, Li S, Yao H, et al. Multiparametric MRI for Bladder Cancer: Validation of VI-RADS for the Detection of Detrusor Muscle Invasion. Radiology. 2019;291(3):668-74.

[7] Takeuchi M, Sasaki S, Ito M, Okada S, Takahashi S, Kawai $\mathrm{T}$, et al. Urinary bladder cancer: diffusion-weighted MR imaging-accuracy for diagnosing $\mathrm{T}$ stage and estimating histologic grade. Radiology. 2009;251(1):112-21.

[8] Yajima S, Yoshida S, Takahara T, Arita Y, Tanaka H, Waseda Y, et al. Usefulness of the inchworm sign on DWI for predicting pT1 bladder cancer progression. Eur Radiol. 2019;29(7): 3881-8.

[9] Kim B, Semelka RC, Ascher SM, Chalpin DB, Carroll PR, Hricak H. Bladder tumor staging: comparison of contrast-enhanced CT, T1- and T2-weighted MR imaging, dynamic gadolinium-enhanced imaging, and late gadolinium-enhanced imaging. Radiology [Internet]. 1994;193(1):239-45. Available from: http://pubs.rsna.org/ doi/10.1148/radiology.193.1.8090898

[10] El-Assmy A, Abou-El-Ghar ME, Refaie HF, Mosbah A, El-Diasty T. Diffusion-weighted magnetic resonance imaging in follow-up of superficial urinary bladder carcinoma after transurethral resection: initial experience. BJU Int [Internet]. 2012;110(11b):E622-7. Available from: http://doi.wiley.com/10.1111/j.1464-410X.2012.11345.x

[11] Sylvester RJ, van der Meijden APM, Oosterlinck W, Witjes JA, Bouffioux C, Denis L, et al. Predicting Recurrence and Progression in Individual Patients with Stage Ta T1 Bladder Cancer Using EORTC Risk Tables: A Combined Analysis of 2596 Patients from Seven EORTC Trials. Eur Urol [Internet]. 2006;49(3):466-77. Available from: https:// linkinghub.elsevier.com/retrieve/pii/S0302283805008523

[12] Fernandez-Gomez J, Madero R, Solsona E, Unda M, Martinez-Piñeiro L, Gonzalez M, et al. Predicting Nonmuscle Invasive Bladder Cancer Recurrence and Progression in Patients Treated With Bacillus Calmette-Guerin: The CUETO Scoring Model. J Urol [Internet]. 2009;182(5): 2195-203. Available from: http://www.jurology.com/doi/ 10.1016/j.juro.2009.07.016
[13] Saito W, Amanuma M, Tanaka J, Heshiki A. Histopathological analysis of a bladder cancer stalk observed on MRI. Magn Reson Imaging. 2000;18(4):411-5.

[14] Ueno Y, Takeuchi M, Tamada T, Sofue K, Takahashi S, Kamishima Y, et al. Diagnostic Accuracy and Interobserver Agreement for the Vesical Imaging-Reporting and Data System for Muscle-invasive Bladder Cancer: A Multireader Validation Study. Eur Urol. 2019;76(1):54-6.

[15] Makboul M, Farghaly S, Abdelkawi IF. Multiparametric MRI in differentiation between muscle invasive and nonmuscle invasive urinary bladder cancer with vesical imaging reporting and data system (VI-RADS) application. Br J Radiol. 2019;92(1104):20190401.

[16] Barchetti G, Simone G, Ceravolo I, Salvo V, Campa R, Del Giudice F, et al. Multiparametric MRI of the bladder: interobserver agreement and accuracy with the Vesical ImagingReporting and Data System (VI-RADS) at a single reference center. Eur Radiol. 2019;29(10):5498-506.

[17] Del Giudice F, Barchetti G, De Berardinis E, Pecoraro M, Salvo V, Simone G, et al. Prospective Assessment of Vesical Imaging Reporting and Data System (VI-RADS) and Its Clinical Impact on the Management of High-risk Nonmuscle-invasive Bladder Cancer Patients Candidate for Repeated Transurethral Resection. Eur Urol. 2020;77(1): $101-9$.

[18] Kim SH. Validation of vesical imaging reporting and data system for assessing muscle invasion in bladder tumor. Abdom Radiol (New York). 2020;45(2):491-8.

[19] Arita Y, Shigeta K, Akita H, Suzuki T, Kufukihara R, Kwee TC, et al. Clinical utility of the Vesical Imaging-Reporting and Data System for muscle-invasive bladder cancer between radiologists and urologists based on multiparametric MRI including 3D FSE T2-weighted acquisitions. Eur Radiol. 2020 Aug; 